\title{
Using Unsteady Aerodynamic Multi-Axis State-Space Formulation as a Tool to represent Wing Rock
}

\author{
Pedro J. De Oliveira-Neto ${ }^{1}$ and William H. Mason ${ }^{2}$ \\ Virginia Polytechnic Institute and State University, Blacksburg, VA 24061 \\ Olympio A. De Faria-Mello ${ }^{3}$ \\ Instituto de Aeronautica e Espaco, Sao Jose dos Campos, SP 12228-904, Brazil
}

\begin{abstract}
A functional unsteady aerodynamic model with state equation representation is proposed and investigated with the purpose of evaluating it as computationally affordable model to be used in conjunction with the equations of motion to simulate wing rock. This state space formulation has three internal state variables that represent parameters related to the physics of the flow. Each airplane lifting surface that the airplane is split into is associated with a set of three state space equations which represents its dynamics, and with one observer equation which outputs its lifting force coefficient. The airplane lifting force coefficient is the summation of the coefficients of each lifting surface panel. The products of the panel force coefficients and their moment arms with reference to the longitudinal axis are summed up to find the global airplane rolling moment coefficient. The proposed airplane state space representation is identified against a combination of experimental aerodynamic data available in the open literature for slender delta wings. The identifications for the proposed formulation are found to match the experimental data well. The simulations in pure roll oscillations revealed that even though it was identified against simulated data based on scarce published experimental results, the model presents the expected qualitative behavior and that the concept is proved to be useful to simulate wing rock.
\end{abstract}

\section{Nomenclature}

$b \quad=$ wing span

$c_{r} \quad=$ wing root chord

$C_{l} \quad=$ roll moment coefficient

$f, g \quad=$ generic functions

Ixx $=$ moment of inertia with respect to the longitudinal axis

$L \quad=$ roll moment

$p \quad=$ roll rate

$\bar{q} \quad=$ dynamic pressure

$S \quad=$ wing planform area

$\phi \quad=$ roll angle

$\tau_{1,(\bullet)} \quad=$ transient time constant

$\tau_{2, \bullet} \quad=$ time-delay constant

\section{Introduction}

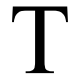

his paper presents a research that is further described in [1]. Its objective is to propose and to investigate an unsteady aerodynamic model with state equation representation and valid up to the high angle of attack regime with the purpose of evaluating it as computationally affordable model to be used in conjunction with the equations

\footnotetext{
${ }^{1}$ Ph.D. candidate, Aerospace and Ocean Engineering Dept., Senior Member AIAA.

${ }^{2}$ Professor, Aerospace and Ocean Engineering Dept., Associate Fellow AIAA.

${ }^{3}$ Senior Research Engineer, Vice-Director for Aeronautics, Senior Member AIAA. 
of motion to simulate wing rock. The aerodynamic forces can be fully described only by the Navier-Stokes equations. At the present time computational methodology and computer power are still not quite adequate to provide time accurate solutions to the Navier-Stokes equations in the Flight Dynamics simulation environment. For this reason, more practical and simpler methods for the determination of the aerodynamic forces that could be used in conjunction with the equations of motion have been searched. These methods can be divided into physical and functional modeling methods. Physical modeling methods for the aerodynamic forces are those directly derived from the very first physical principles through the simplifications of the Navier-Stokes equations. The multi-axis model $^{2}$ chosen in this report to represent the unsteady aerodynamics of the whole airplane assumes the decomposition of the airplane into lifting surfaces or panels that have their particular aerodynamic force coefficients modeled as basic unsteady aerodynamic state-space models ${ }^{3}$. These coefficients are summed up to find the total aircraft force coefficients. The products of the panel force coefficients and their moment arms with reference to a given axis are summed up to find the global aircraft moment coefficients. The state equations formulation proposed here to represent the basic unsteady aerodynamic model of each single lifting surface are functional approaches to modeling aerodynamic phenomena, not directly derived from the physical principles of the problem. It is thought to have advantages with respect to the physical modeling methods mainly because of its lower computational cost involved in the calculations while still keeping some ability to represent the dynamic behavior. It has advantages over the previous functional, state space formulations because of its three internal state variables that allow a better consistency with the physics of the flow.

\section{Proposed Unsteady Aerodynamic Formulation}

For the wing rock limit-cycle oscillations to happen, the rolling moment coefficient must laterally destabilize the wing at small roll angles and stabilize it at their bigger values ${ }^{4}$. The fluid mechanisms that do it and sustain the limit-cycle oscillations involve time lag in vortex core position and strength ${ }^{5}$. In the present model, we propose to match these effects with a basic unsteady aerodynamic model that represents the variation of the panel normal lifting forces with the strength and vertical position of the vortex core vertical position in function of the panel roll angle. Therefore, in this formulation, the panel normal lifting forces are modeled to be functions of both the angle of attack and the roll angle. Since the wing rock oscillations of this type of configuration are strongly related to the movement of the leading edge vortices, we conceive these functions by assuming that the spanwise positions of the points of application of the panel normal lifting forces coincide with the vortex core positions. Therefore, the spanwise nondimensional body-axes coordinates $\bar{y}_{i}(t)=y_{i}(t) / b$ are also taken as internal flow state variables, in addition to the chordwise coordinates $\bar{x}_{i}(t)=x_{i}(t) / c_{r}$, which are associated to the region of the vortex breakdown ahead of the panel leading edge, as it is shown in Figure 1. A third type $\bar{v}_{i}(t)$ of state variable, associated to the effects of both the vortex strength and the vortex core vertical position $Z_{i}(t)$, is still added to the system. Therefore, the basic unsteady aerodynamic model proposed here is, in its most general form, composed by the state equations of the above mentioned state variables and the observer equations that outputs the values of the airplane rolling moment and normal force coefficients. The airplane force coefficients are the summation of the coefficients of each lifting surface panel that the airplane is split into. The state equations are the first-order differential equations (1) to (3), whose above mentioned dependent variables $\bar{x}_{i}(t), \bar{y}_{i}(t)$, and $\bar{v}_{i}(t)$ represent the system dynamics. Equations (4) and (5) are the output equations. The input variables are the kinematic motion parameters, also called motion variables: the panel local angle of attack $\alpha_{i}(t)$, the airplane roll angle $\phi(t)$, and their respective time derivatives $\dot{\alpha}_{i}(t), \dot{\phi}(t)$. For each fixed value of the wing pitch angle $\theta_{0}$, the local sideslip angle time history $\beta_{i}(t)$ is promptly determined through $\phi(t)$ time-history by using Eq. (8).

In this postulated model, the equations used to represent the unsteady aerodynamics for general flight conditions in each panel of the airplane are:

$$
\frac{d \bar{x}_{i}(t)}{d t}=f_{1, i}\left(\alpha_{i}, \dot{\alpha}_{i}\right)
$$




$$
\begin{aligned}
& \frac{d \bar{y}_{i}(t)}{d t}=f_{2, i}(\phi, \dot{\phi}) \\
& \frac{d \bar{v}_{i}(t)}{d t}=f_{3, i}(\phi, \dot{\phi}) \\
& C_{N, i}=g\left(\bar{x}_{i}, \bar{y}_{i}, \alpha_{i}, \dot{\alpha}_{i}, \phi, \dot{\phi}\right)
\end{aligned}
$$

where the subscript $i$ stands for the considered lifting panel, i.e., $i=l$ (left) $r$ (right).

The value of the normal force coefficient for the whole slender delta wing is found through Eq. (5).

$$
C_{N}=C_{N, l}+C_{N, r}
$$

The rolling moment coefficient is determined through Eq. (6),

$$
C_{l}=C_{N l}\left|\bar{y}_{l}(\phi)\right|-C_{N r}\left|\bar{y}_{r}(\phi)\right|
$$

where $\bar{y}_{(\bullet)}$ are the non-dimensional arms of the normal force coefficient with respect to the longitudinal body axis, whose dynamic behavior is given by Eq. (10).

Aerodynamic angles at each wing panel are determined by the equations

$$
\begin{aligned}
& \alpha_{i}=\tan ^{-1}\left(\tan \theta_{0} \cos \phi-\frac{l_{i} \dot{\phi}}{V \cos \theta_{0}}\right) \\
& \beta_{i}=\sin ^{-1}\left(\sin \theta_{0} \sin \phi+\frac{l_{i} \dot{\phi}}{V}\right)
\end{aligned}
$$

being $l_{i}$ the distance to the rotation axis where the local aerodynamic angle must be determined, and $i=l, r$.

With the help of the formulation described in Ref. [6], the system composed by the Eqs. (1) to (4) is unfolded to Eqs. (9) to (12):

$$
\begin{aligned}
& \tau_{1 x} \frac{d \bar{x}_{i}}{d t}+\bar{x}_{i}=U_{\alpha} \bar{x}_{0, U}\left(\alpha_{e f f, i}\right)+D_{\alpha} \bar{x}_{0, D}\left(\alpha_{e f f, i}\right) \\
& \tau_{1 y} \frac{d \bar{y}_{i}}{d t}+\bar{y}_{i}=U_{\phi} \bar{y}_{0, i, U}\left(\phi_{e f f}\right)+D_{\phi} \bar{y}_{0, i, D}\left(\phi_{e f f}\right) \\
& \tau_{1 v} \frac{d \bar{v}_{i}}{d t}+\bar{v}_{i}=U_{\phi} \bar{v}_{0, i, U}\left(\phi_{e f f}\right)+D_{\phi} \bar{v}_{0, i, D}\left(\phi_{e f f}\right) \\
& C_{N, i}=C_{N, i}\left(\bar{x}_{i}, \alpha_{i}\right)+\Delta C_{N, i}\left(\bar{v}_{i}, \phi\right)
\end{aligned}
$$

On the right hand side of the above equations are the functions that represent the static dependencies. They are written this way because we want this model capable of representing also static hysteresis, whenever it occurs. In 
Eq. (9), we have that $\tau_{1, x}$ is the transient-time-constant associated with the displacement of the vortex breakdown chordwise position, and:

$$
\begin{gathered}
U_{\alpha} \stackrel{\Delta}{=} \frac{1+\operatorname{sign}\left(\Delta \alpha_{i}\right)}{2} \\
D_{\alpha}=\frac{\Delta-\operatorname{sign}\left(\Delta \alpha_{i}\right)}{2}
\end{gathered}
$$

with $\Delta \alpha_{i}=\alpha_{i, j+1}-\alpha_{i, j}$ for the given sequence of the static angles of attack $\alpha_{i, j}, i=l, r ; j=1,2, \ldots, l$, or $\Delta \alpha_{i}=\alpha_{i}\left(t_{j+1}\right)-\alpha_{i}\left(t_{j}\right)$ for the given time histories of angles of attack $\left\{\alpha_{i}\left(t_{j}\right), 0 \leq t_{j} \leq t_{n}\right\}$ in the considered panel. The static dependence between the internal state variable $\bar{x}_{i}$ and the angle of attack are determined for the up $(U)$ and down $(D)$ direction by

$$
\begin{aligned}
& \bar{x}_{0 U}=\frac{1}{1+\exp \left[-\sigma_{x, U}\left(\alpha_{e f f, i}-\alpha_{U}^{*}\right)\right]} \\
& \bar{x}_{0 D}=\frac{1}{1+\exp \left[-\sigma_{x, D}\left(\alpha_{e f f, i}-\alpha_{D}^{*}\right)\right]}
\end{aligned}
$$

Since all the effects due to the sideslip or to the roll angle are included in term $\Delta C_{N, i}(\phi)$ of Eq. (12), the parameters $\alpha_{(\bullet)}^{*}$ and $\sigma_{x,(\bullet)}$ related to the localization and shape of the sigmoids given by Eqs. (15) and (16) are not let free to vary with the roll angle. The values of $\alpha_{\text {eff }}$ are determined through the equation

$$
\alpha_{e f f, i}=\alpha_{i}-\tau_{2, \alpha} \dot{\alpha}_{i}
$$

with $\tau_{2, \alpha}$ being a time-delay-constant related to vortex burst location. Equation (10) is similar in structure to (9), and its nomenclature is given as follows,

$\tau_{1 y}=$ the transient-time-constant related to the vortices core spanwise displacement.

$\bar{y}_{i}=\frac{y_{i}}{b}=$ non-dimensional distance between the panel normal lifting force point of application and the longitudinal axis.

$$
\begin{gathered}
U_{\phi} \stackrel{\Delta}{=} \frac{1+\operatorname{sign}(\Delta \phi)}{2} \\
D_{\phi} \stackrel{\Delta}{=} \frac{1-\operatorname{sign}(\Delta \phi)}{2}
\end{gathered}
$$

with $\Delta \phi=\phi_{j+1}-\phi_{j}$ or $\Delta \phi=\phi\left(t_{j+1}\right)-\phi\left(t_{j}\right)$ respectively in the quasi-static and in the dynamic cases, where, in the quasi-static case, the given sequence of the static roll angles is $\phi_{j}, j=1,2, \ldots, l$, and where $\Delta \phi=\phi\left(t_{i+1}\right)-\phi\left(t_{i}\right)$ 
for the given time histories of roll angles $\left\{\phi\left(t_{i}\right), 0 \leq t_{i} \leq t_{n}\right\}$. The time-delay effects on the vortex movements due to the roll angle are taken into account through

$$
\phi_{\text {eff }}=\phi-\tau_{2, \phi} \dot{\phi}
$$

where $\tau_{2, \phi}$ is a time-delay constant related to the vortex core position, to be identified against wind tunnel data.

Since vortex strength and vertical core position effects are considered to be lumped into Eq. (11), we want the state variables $\bar{y}_{i}$ behaving qualitatively like in the wind tunnel tests results shown on Ref. [5], what means that $\bar{y}_{i}$ must get smaller for the panel that is going down. Because of that, the logistic functions that compose the right-hand-side of Eq. (10) are built as follows,

$$
\begin{aligned}
& \bar{y}_{0, i, U}(\phi)=\frac{1}{1+\exp \left[\xi_{i} \sigma_{y, i, U}\left(\phi-\phi_{y, i, U}^{*}\right)\right]} \\
& \bar{y}_{0, i, D}(\phi)=\frac{1}{1+\exp \left[\xi_{i} \sigma_{y, i, D}\left(\phi-\phi_{y, i, D}^{*}\right)\right]}
\end{aligned}
$$

where $\xi_{l}=-1$ and $\xi_{r}=1$ respectively for left $(i=l)$ and right $(i=r)$ panels. Symbols $\sigma_{y, i,(\bullet)}$ and $\phi_{y, i,(\bullet)}^{*}$ stand respectively for the slope and roll angle location of the logistic functions that represent the static variation of the vortex core spanwise position with the roll angle.

The state variable $\bar{v}$ is conceived to represent the increase in panel lift due to both the increase in vortex strength and the approximation of the vortex core to the panel upper surface, as the wing rolls. Because of that, the forcing terms of on the right-hand side of Eq. (11) are modeled to be

$$
\begin{aligned}
& \bar{v}_{0, i, U}(\phi)=\frac{1}{1+\exp \left[-\xi_{i} \sigma_{v, i, U}\left(\phi-\phi_{v, i, U}^{*}\right)\right]} \\
& \bar{v}_{0, i, D}(\phi)=\frac{1}{1+\exp \left[-\xi_{i} \sigma_{v, i, D}\left(\phi-\phi_{v, i, D}^{*}\right)\right]}
\end{aligned}
$$

In these last equations, symbols $\sigma_{v, i,(\bullet)}$ and $\phi_{v, i,(\bullet)}^{*}$ stand respectively for the slope and roll angle location of the logistic functions that represent the static variation of the effects of vortex vertical position and strength with the roll angle.

In Eq. (12), the first parcel $C_{N, i}\left(\bar{x}, \alpha_{i}\right)$ is taken as it was developed in Ref. [7], that is, as an expansion in terms of the angle of attack $\alpha$ and its non-dimensional time-derivative $\hat{\dot{\alpha}}$, for the roll angle equal zero:

$$
C_{N, i}=C_{N 0}+C_{N \alpha}\left(\bar{x}_{i}\right) \alpha_{i}+C_{N \alpha^{2}}\left(\bar{x}_{i}\right) \alpha_{i}^{2}+C_{N \hat{\alpha}}\left(\bar{x}_{i}\right) \hat{\dot{\alpha}}_{i}+C_{N \hat{\dot{\alpha}}^{2}} \hat{\dot{\alpha}}_{i}^{2}+C_{N \alpha \hat{\alpha}}\left(\bar{x}_{i}\right) \alpha_{i} \hat{\dot{\alpha}}_{i}
$$

where

$$
C_{N_{\chi}}\left(\bar{x}_{i}\right)=a_{j}+b_{j} \bar{x}_{i}+c_{j} \bar{x}_{i}^{2}
$$


with $\chi=\alpha, \alpha^{2}, \hat{\dot{\alpha}}, \hat{\dot{\alpha}}^{2}, \alpha \hat{\dot{\alpha}}$, and $j=1,2,3,4,5$, respectively. Parameters $a_{j}, b_{j}, c_{j}$ are constants to be identified.

The motion variable $\hat{\dot{\alpha}}=\hat{t} \dot{\alpha}$ is the non-dimensional angle of attack rate and $\hat{t}$ is the characteristic time of the flow defined as:

$\hat{t}=\frac{C}{2 V}$

In Eq. (12), $\Delta C_{N, i}(\phi)$ is the parcel that accounts for the effects of the roll angle at a fixed pitch angle value $\theta_{0}$. It is this normal force term that accounts for the effects due to both vortex vertical position and strength. The normal lifting force in a panel gets bigger as the roll angle changes in a way that makes the panel to go down. Also, limitcycle oscillations are sustained by a time lag in vortex core position and strength. In order to make this model capable of handling these variations in a nonlinear way, the panel normal force parcel $\Delta C_{N, i}(\phi)$ is built according to the following dependence of the roll angle $\phi$ and of the non-dimensional roll rate $\hat{\dot{\phi}}$ :

$$
\begin{aligned}
& \Delta C_{N, i}\left(\bar{v}_{i}, \phi\right)=C_{N \phi}\left(\bar{v}_{i}\right) \phi+C_{N \phi^{3}}\left(\bar{v}_{i}\right) \phi^{3}+C_{N \phi^{5}}\left(\bar{v}_{i}\right) \phi^{5}+C_{N \dot{\phi}}\left(\bar{v}_{i}\right) \hat{\dot{\phi}}+C_{N \phi^{3}}\left(\bar{v}_{i}\right) \hat{\dot{\phi}}^{3} \\
& +C_{N \phi^{2} \dot{\phi}}\left(\bar{v}_{i}\right) \phi^{2} \hat{\dot{\phi}}+C_{N \phi \phi^{2}}\left(\bar{v}_{i}\right) \phi \hat{\phi}^{2}
\end{aligned}
$$

where

$$
\begin{aligned}
& \hat{\dot{\phi}}=\dot{\phi} \frac{b}{2 V} \\
& C_{N_{\chi}}\left(\bar{v}_{i}\right)=a_{j}+b_{j} \bar{v}_{i}+c_{j} \bar{v}_{i}^{2}
\end{aligned}
$$

with

$\chi=\phi, \phi^{3}, \phi^{5}, \dot{\phi}, \dot{\phi}^{3}, \phi^{2} \dot{\phi}, \phi \dot{\phi}^{2}$, and $j=6,7,8,9,10,11,12$.

$V=$ free stream airspeed.

The coefficients $a_{j}, b_{j}, c_{j}$ here are functions of the pitch angle $\theta_{0}$, and are determined by a least-squares fit with the experimental data. The expansion terms in Eq. (28) were picked in order to match the expansion developed by Konstadinopoulos, Mook, and Nayfeh ${ }^{8}$ for $C_{l}(\phi)$.

The 62 grand-total of parameters to be identified against wind tunnel data for this postulated model are dependent on the pitch angle $\theta_{0}$ at which the identification is done. They are:

- the polynomial coefficients $C_{N o}, a_{j}, b_{j}, c_{j}, j=1,2,3,4,5$ of Eqs. (25) and (26);

- the polynomial coefficients $a_{j}, b_{j}, c_{j}, j=6,7,8,9,10,11,12$ of Eqs. (28) and (30);

- transient-time constants $\tau_{1 x}, \tau_{1 y}, \tau_{1 v}$ appearing respectively in Eqs. (9), (10), and (11);

- time-delay constants $\tau_{2, \alpha}, \tau_{2, \phi}$ from Eqs. (17) and (20);

- parameters $\alpha_{(\bullet)}^{*}, \phi_{(\bullet)}^{*}$, and $\sigma_{(\bullet)}$ of logistic equations (15), (16), (21), (22), (23), and (24). 


\section{Parameter Identification Method}

The identification method used here is the minimum mean-square error approach. The models are identified against experimental data in three phases and the squared error is minimized through the multi-objective optimization process. In the two first phases, the parameter identification is done against static experimental data. First, we identify the parameters involved with the determination of the static terms of the normal lifting forces $\left(\vec{x}_{C N}\right)$. These parameters are those related to the quadratic polynomials that determine the stability derivatives with respect to the motion variables $\left(a_{j}, b_{j}, c_{j}\right)$, and those that are used to determine the static values of the internal state variables $\bar{X}_{i}$, namely $\alpha_{(\bullet)}^{*}, \sigma_{(\bullet)}$. Next, we identify only the parameters associated to the arms of the rolling moment coefficients $\left(\vec{x}_{C l}\right)$ against the experimental static values of the rolling moment coefficient. In those two first phases of the parameter identification, the parameters related to the dynamic behavior of the model are kept fixed. These dynamic parameters are the coefficients of the quadratic polynomials that represent the stability derivatives with respect to the time-rate of the motion variables, and the time-constant and time-delay parameters, all of them stored in vector $\vec{x}_{d y n}$. The dynamic phase of the identification is done against the experimental values of the rolling moment time history.

When the quasi-static sequences of experimental data are considered, the error cost-functions to be minimized are as follows,

$$
\begin{aligned}
& \varepsilon_{C N}=\frac{1}{I+J} \sum_{j=1}^{N} \sum_{i=1}^{M}\left[\hat{C}_{N}\left(\theta_{0 i}, \phi_{j}\right)-C_{N}\left(\theta_{0 i}, \phi_{j}\right)\right]^{2} \\
& \varepsilon_{C l}=\frac{1}{M+N} \sum_{j=1}^{N} \sum_{i=1}^{M}\left[\hat{C}_{l}\left(\theta_{0 i}, \phi_{j}\right)-C_{l}\left(\theta_{0 i}, \phi_{j}\right)\right]^{2}
\end{aligned}
$$

where each value of the roll angle in the sequence $\phi_{j}, j=1,2, \ldots, N$ has a correspondent sequence of values for the wind tunnel sting pitch angle $\theta_{0 i}, i=1,2, \ldots, M$. At these points, the wind tunnel measurements are $\left\{\hat{C}_{N}\left(\theta_{0 i}, \phi_{j}\right), \hat{C}_{l}\left(\theta_{0 i}, \phi_{j}\right) ; i=1,2, \ldots, M ; j=1,2, \ldots, N\right\}$, while the model responses determined at the same points for a given set of model parameters are $\left\{C_{N}\left(\theta_{0 i}, \phi_{j}\right), C_{l}\left(\theta_{0 i}, \phi_{j}\right), i=1,2, \ldots, M ; j=1,2, \ldots, N\right\}$.

When the published values of wind tunnel dynamic measurements at unsteady flow conditions are used to identify the remaining parameters, one roll angle time history $\left\{\phi\left(t_{i}\right), 0 \leq t_{i} \leq T_{F}\right\}$, such that $0=t_{1}<t_{2}<\ldots<t_{i}<t_{i+1}<\ldots<t_{L}=T_{F}$, is taken for each fixed value of the sting pitch angle $\theta_{0}$ in the sequence $\theta_{0 k}, k=1,2, \ldots, K$. If the corresponding dynamic wind tunnel measurements of the rolling moment coefficient can be represented by $\left\{\hat{C}_{l}\left(\phi\left(t_{i}, \theta_{0 k}\right)\right) ; i=1,2, \ldots, L ; k=1,2, \ldots, K\right\}$ and the values of the model responses calculated at the same points are $\left\{C_{l}\left(\phi\left(t_{i}, \theta_{0 k}\right)\right) ; i=1,2, \ldots, L ; k=1,2, \ldots, K\right\}$, the error cost-function for the dynamic phase of the identification is

$$
\varepsilon_{d y n}=\frac{1}{L+K} \sum_{k=1}^{K} \sum_{i=1}^{L}\left[\hat{C}_{l}\left(\phi\left(t_{i}, \theta_{0 k}\right)\right)-C_{l}\left(\phi\left(t_{i}, \theta_{0 k}\right)\right)\right]^{2}
$$

For known sequences of experimental data, the design variables in the cost-functions (31) to (33) are the model parameters stored in $\vec{x}_{C N}, \vec{x}_{C l}$, and $\vec{x}_{d y n}$. Representing the cost-functions in terms of these model parameters, we

have $f_{C N}\left(\vec{x}_{C N}\right), f_{C l}\left(\vec{x}_{C l}\right)$, and $f_{d y n}\left(\vec{x}_{d y n}\right)$. In this particular case, these individual cost-functions are controlled 
by different sets of variables, that is, $\vec{x}_{C N}^{*}, \vec{x}_{C l}^{*}, \vec{x}_{d y n}^{*}$ are the solutions to individual objectives, and $\vec{x}_{C N}^{*} \neq \vec{X}_{C l}^{*} \neq \vec{X}_{d y n}^{*}$. Then, the optimum of

$F(\vec{x})=f_{C N}\left(\vec{x}_{C N}\right)+f_{C l}\left(\vec{x}_{C l}\right)+f_{d y n}\left(\vec{x}_{d y n}\right)$

can be obtained by optimizing the individual $f_{(\bullet)}$ 's, that is,

$$
\begin{aligned}
& \left(\vec{x}_{C N}^{*}, f_{C N}^{*}\right)=\min f_{C N}(\vec{x}) \\
& \left(\vec{x}_{C l}^{*}, f_{C l}^{*}\right)=\min f_{C l}(\vec{x}) \\
& \left(\vec{x}_{d y n}^{*}, f_{d y n}^{*}\right)=\min f_{d y n}(\vec{x})
\end{aligned}
$$

where $\vec{x}=\left[\begin{array}{lll}\vec{x}_{C N} & \vec{x}_{C l} & \vec{x}_{d y n}\end{array}\right]^{T}$

\section{Numerical Simulations in Roll}

In this Section, the single degree of freedom numerical simulation in roll of a slender delta wing with aspect ratio equal 0.71 taken from Ref. [9] is carried out by using the unsteady aerodynamic model described above and identified against simulated experimental data composed by a combination of experimental data extracted from references [3], [4], and [9].

For the single degree of freedom in roll, the rigid body 6 DOF system of equations is simplified to

$I_{x x} \dot{p}=L$

$p=\dot{\phi}$

In order to describe the complete dynamic system, the rigid body system of equations must be integrated to the unsteady aerodynamic model equations. The aerodynamic model comes to the rigid body equations (36) through the rolling moment coefficient, that is:

$\dot{p}=\ddot{\phi}=\frac{\bar{q} S b}{I_{x x}} C_{l}$

As it can be seen in Eq. (6), for the proposed aircraft unsteady aerodynamic model the moment coefficients are obtained by multiplying left and right wing force coefficients by their arms along the corresponding body axis. In order to determine the delta wing motion history, Eqs. (36) are coupled to Eqs. (9), (10), and (11). The dynamic system built this way therefore is composed by the following eight first-order differential equations:

$$
\begin{aligned}
& \dot{\phi}=p \\
& \dot{p}=\frac{\bar{q} S b}{I_{x x}} C_{l}
\end{aligned}
$$




$$
\begin{gathered}
\tau_{1 x} \frac{d \bar{x}_{l}}{d t}+\bar{x}_{l}=U_{\alpha} \bar{x}_{0, U}\left(\alpha_{e f f, l}\right)+D_{\alpha} \bar{x}_{0, D}\left(\alpha_{e f f, l}\right) \\
\tau_{1 x} \frac{d \bar{x}_{r}}{d t}+\bar{x}_{r}=U_{\alpha} \bar{x}_{0, U}\left(\alpha_{e f f, r}\right)+D_{\alpha} \bar{x}_{0, D}\left(\alpha_{e f f, r}\right) \\
\tau_{1 y} \frac{d \bar{y}_{l}}{d t}+\bar{y}_{l}=U_{\phi} \bar{y}_{0, l, U}\left(\phi_{e f f}\right)+D_{\phi} \bar{y}_{0, l, D}\left(\phi_{e f f}\right) \\
\tau_{1 y} \frac{d \bar{y}_{r}}{d t}+\bar{y}_{r}=U_{\phi} \bar{y}_{0, r, U}\left(\phi_{e f f}\right)+D_{\phi} \bar{y}_{0, r, D}\left(\phi_{e f f}\right) \\
\tau_{1 v} \frac{d \bar{v}_{l}}{d t}+\bar{v}_{l}=U_{\phi} \bar{v}_{0, l, U}\left(\phi_{e f f}\right)+D_{\phi} \bar{v}_{0, l, D}\left(\phi_{e f f}\right) \\
\tau_{1 v} \frac{d \bar{v}_{r}}{d t}+\bar{v}_{r}=U_{\phi} \bar{v}_{0, r, U}\left(\phi_{e f f}\right)+D_{\phi} \bar{v}_{0, r, D}\left(\phi_{e f f}\right)
\end{gathered}
$$

All the dynamic equations (38) and (39) must be integrated simultaneously in order to simulate the slender delta wing pure roll motion. The internal state variables $\bar{x}_{i}, \bar{y}_{i}, \bar{v}_{i}$ are coupled to the two first equations of motion through the roll moment coefficient $C_{l}$, which is determined by Eq. (6). In that equation, the left and right panel normal force coefficients are multiplied by their respective arms $\bar{y}_{i}$. The normal force coefficients are calculated from the remaining internal state variables through Eqs. (12), (25), (26), (28), and (30).

\section{Results}

\section{A. Identifications Results}

Previously published experimental data are combined and used to identify the parameters of the investigated models. The static data used for the parameter identification are those obtained by Levin and $\mathrm{Katz}^{9}$ for their slender delta wing model of aspect ratio equal 0.71 . Here, we take that wing and assume that it undergoes wing rock for pitch angles in between 22 and 45 degree. It is also admitted to have dynamic behavior during steady state limit cycles similar to experimental data obtained by Nguyen, Yip, and Chambers ${ }^{4}$ for $\theta_{0}=27$ and $32 \mathrm{deg}$. The roll moment coefficient experimental histogram for $\theta_{0}=27 \mathrm{deg}$ is also used to identify the model parameters for $\theta_{0}=$ $38 \mathrm{deg}$. For the wing pitch angles at which the oscillations in roll damp out, the experimental data used to identify the parameters are assumed to have the same qualitative behavior exhibited on Figures 2 and 5. Figures 3 and 4 illustrate situations in which the wing undergoes limit-cycles oscillations. According to the Energy Exchange Concept ${ }^{4}$, the limit-cycles oscillations exist because in each cycle dissipated energy equals energy received from the fluid.

\section{B. Simulations Results}

The mixed characteristics of the wing used in the simulations are span $b=0.150 \mathrm{~m}$, root chord $c_{r}=0.4285 \mathrm{~m}$, sweep angle at the leading edge 80 degree, and rolling moment of inertia $I_{x x}=9.18 \times 10^{-2} \mathrm{~kg} \mathrm{~m}^{2}$. The air density value adopted for the simulations is $\rho=1.2 \mathrm{~kg} / \mathrm{m}^{3}$, and the airspeed is $V=9.27 \mathrm{~m} / \mathrm{s}$. The first simulation is done for the wing starting from rest at $\phi_{0}=5 \mathrm{deg}, \theta_{0}=30 \mathrm{deg}$. At these conditions, the wing is expected to exhibit wing 
rock, what effectively happens, as Figures 5 and 6 show. The roll attractor is not located at null roll angle, as it was expected from wind tunnel tests like the one whose results are shown on [4]. Also, the amplitude is smaller in the present simulations. We attribute this and other differences between these simulations and the classical wing rock behavior to the scarcity of the published experimental data so far used to identify the parameters. Figures 7 and 8 show the results of the simulation starting at $\theta_{0}=30 \mathrm{deg}, \phi_{0}=-5 \mathrm{deg}$, and $\dot{\phi}_{0}=0 \mathrm{deg} / \mathrm{s}$, where we can see that the roll attractor at $\phi=-12 \mathrm{deg}$ is confirmed. To compare better our results to those shown on [4], we simulate the wing rock with the wing departing from the roll attractor position. The results can be seen on Figures 9 and 10. They show that the build up time is much shorter in the present case.

\section{Concluding Remarks}

By following the guidance of physical properties of the flow observed in previous works, it is proposed a statespace system capable of simulating the wing rock dynamics. In the presented case, a delta wing configuration is split in two panels, and the simulation is carried out for just one degree of freedom. The resulting dynamic system contains two equations of motion plus six internal state equations for the unsteady aerodynamic model, making a grand total of eight first-order differential equations to be simultaneously integrated, which is easier to do than to use a physical method. The simulations in pure roll oscillations revealed that even though it was identified against simulated data based on scarce published experimental results, the model presents the expected qualitative behavior and that the concept is proved as useful to simulate wing rock.

\section{Acknowledgments}

This research was funded by FUNCATE, a Brazilian research agency, and by the Brazilian Air Force's Institute of Aeronautics and Space (IAE).

\section{References}

${ }^{1}$ De Oliveira-Neto, P. J., “An Investigation of Unsteady Aerodynamic Multi-Axis State-Space Formulations as a Tool for Wing Rock Representation," Ph. D. Dissertation, Aerospace and Ocean Engineering Dept., Virginia Polytechnic Institute and State University, Blacksburg, VA ( to be published ).

${ }^{2}$ Lutze, F. H., Fan, Y. G., and Stagg, G., "Multiaxis Unsteady Aerodynamic Characteristcs of an Aircraft," AIAA Atmospheric Flight Mechanics Conference and Exhibit, Portland, OR, Aug. 9-11, 1999, Collection of Technical Papers (A9936864 09-08).

${ }^{3}$ Goman, M., and Khrabrov, A. N., "State-space Representation of Aerodynamic Characteristics of an Aircraft at High Angles of Attack," Journal of Aircraft, Vol. 31, No. 5, Sept.-Oct. 1994, pp. 1109-1115.

${ }^{4}$ Nguyen, L. T., Yip L., Chambers J. R., "Self-Induced Wing Rock of Slender Delta Wings," AIAA Paper 81-1883, AIAA AFM Conference, Albuquerque, New Mexico, August 1981.

${ }^{5}$ Arena Jr. A., “An Experimental and Computational Investigation of Slender Wings Undergoing Wing Rock.," Ph.D. Dissertation, University of Notre Dame, Notre Dame, IN, April 1992.

${ }^{6}$ De Oliveira-Neto, P. J., Lutze, F. H., "First-Order Unsteady Aerodynamic Model that Includes the Static Hysteresis Penomenon," AIAA-2002-4083, Monterey, CA, 2002

${ }^{7}$ Fan, Y., Lutze, F. H., "Identification of an Unsteady Aerodynamic Model at High Angles of Attack," AIAA Paper 96-3407, 1996.

${ }^{8}$ Konstadinopoulos, P., Mook, D. T., Nayfeh, A. H., "Subsonic Wing Rock of Slender Delta Wings,” Journal of Aircraft, Vol 22, No. 3, March 1985, pp. 223-228.

${ }^{9}$ Levin, D., Katz, J., "Dynamic Load Measurements with Delta Wings Undergoing Self-Induced Roll Oscillations," Journal of Aircraft, Vol. 21, No. 1, pp. 30-36, 1984. 

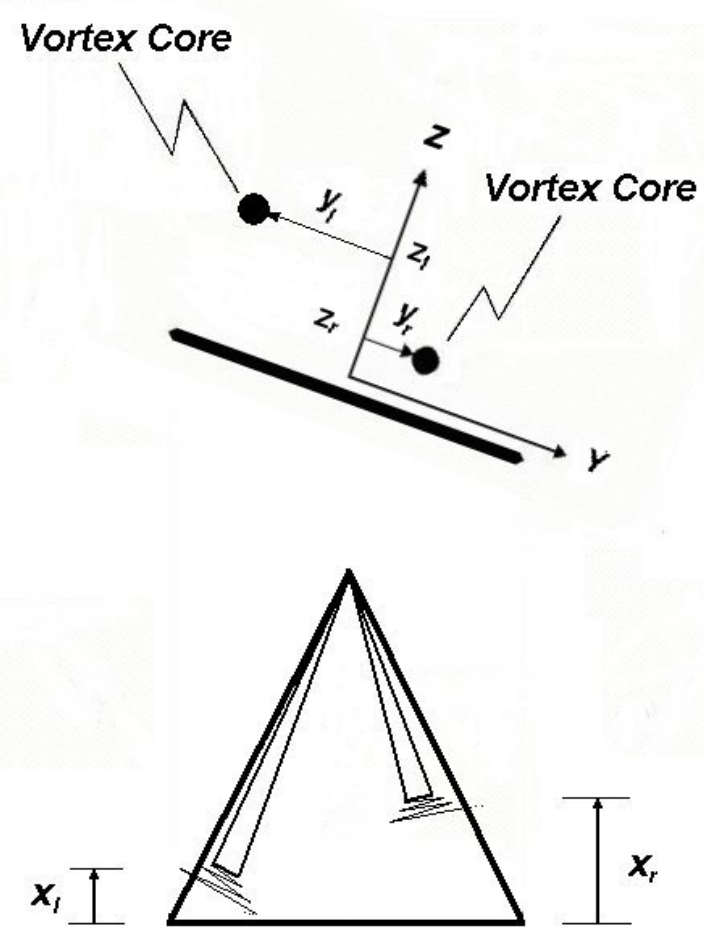

Figure 1. Sketch of asymmetric vortex position system.

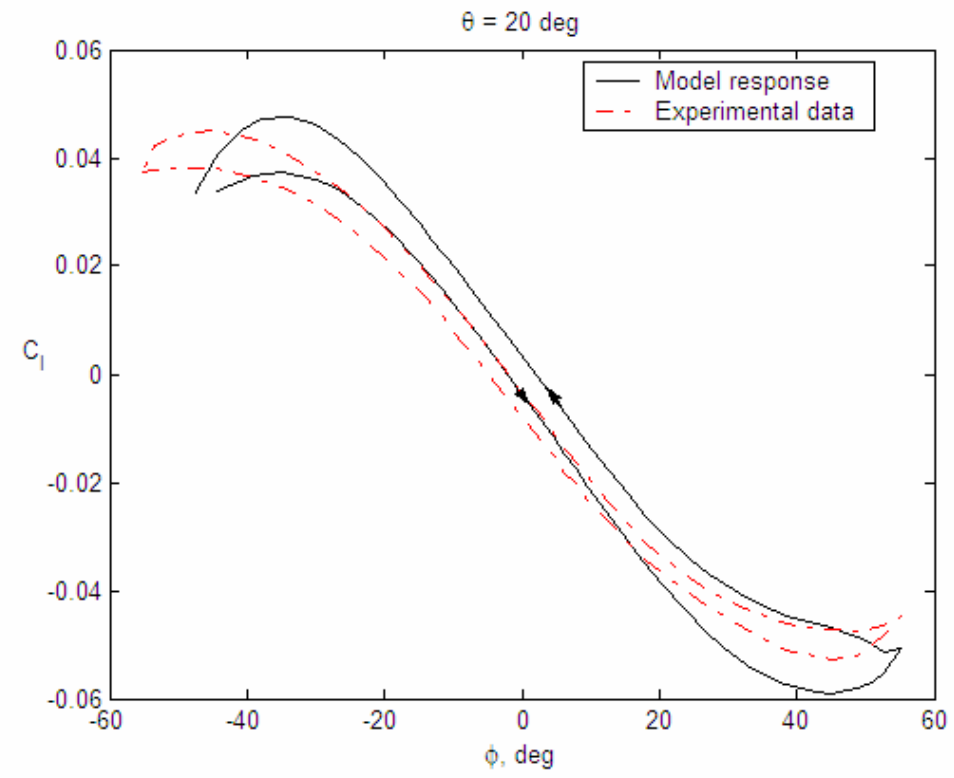

Figure 2. Rolling moment coefficient vs. roll angle loops at $\theta_{0}=20 \mathrm{deg}$. 


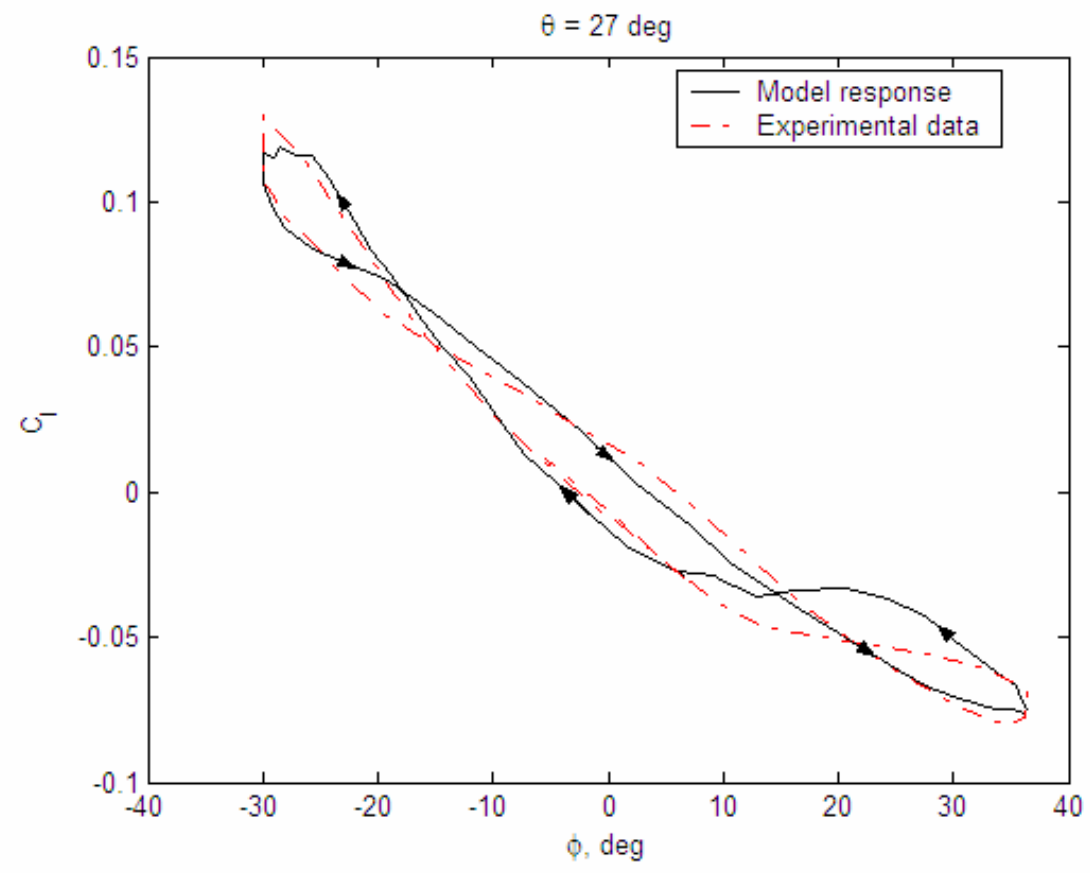

Figure 3. Rolling moment coefficient vs. roll angle loops at $\theta_{0}=27 \mathrm{deg}$.

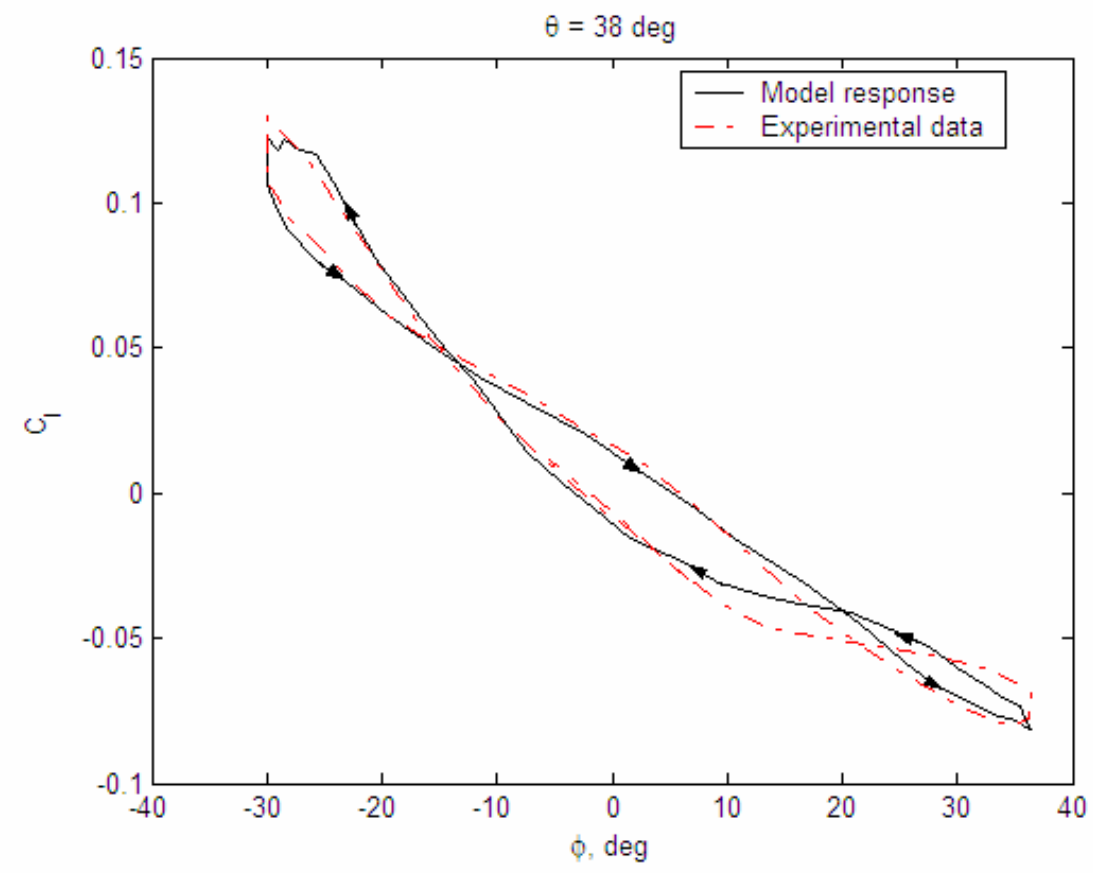

Figure 4. Rolling moment coefficient vs. roll angle loops at $\theta_{0}=38 \mathrm{deg}$. 


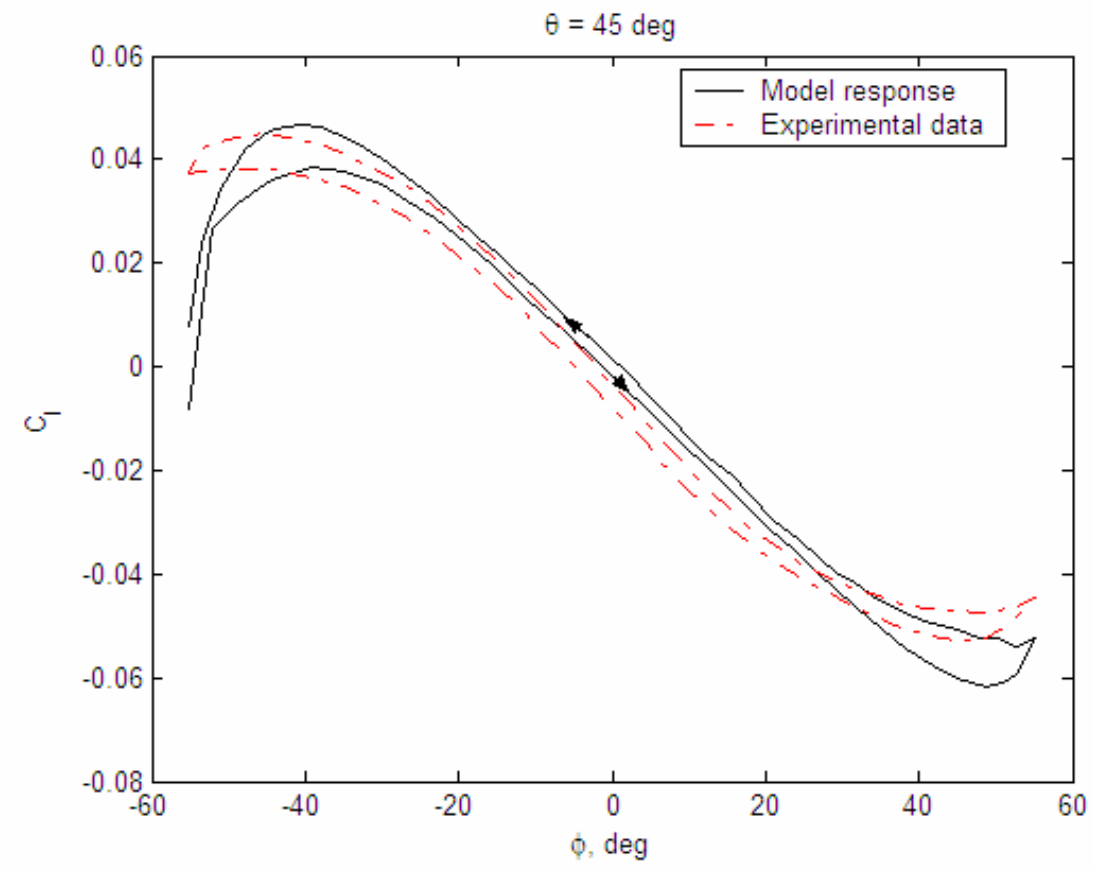

Figure 5. Rolling moment coefficient vs. roll angle loops at $\theta_{0}=45 \mathrm{deg}$

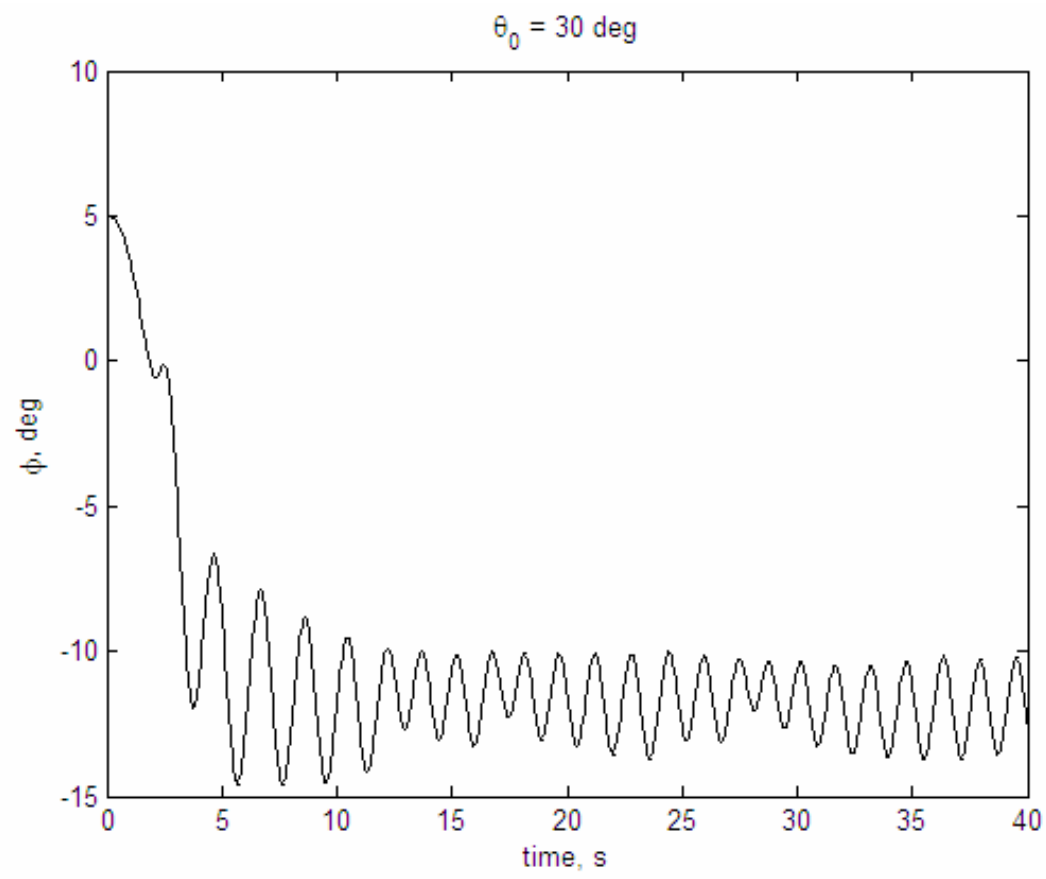

Figure 6. Free to roll simulations at wing pitch angle equal $30 \mathrm{deg}$, starting at roll angle equal 5 degree. 


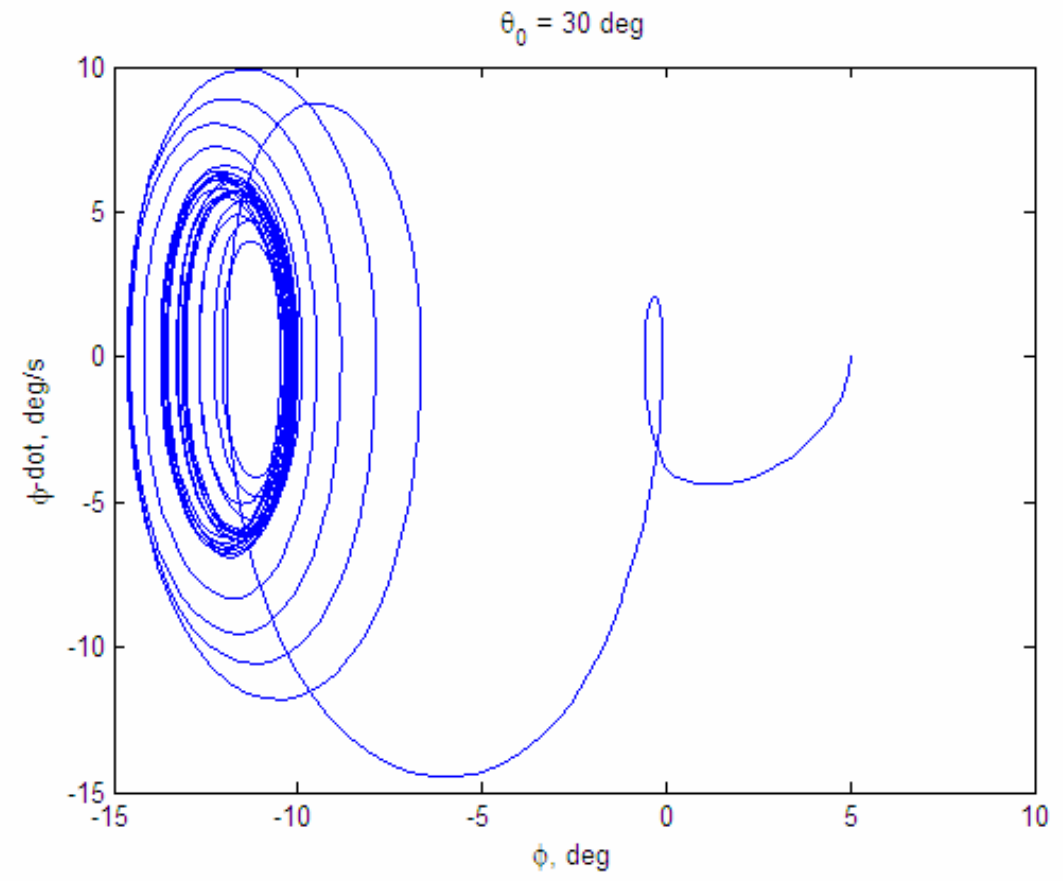

Figure 7. Phase-plane of the simulation response at pitch angle equal $30 \mathrm{deg}$, initial roll angle of 5 degree.

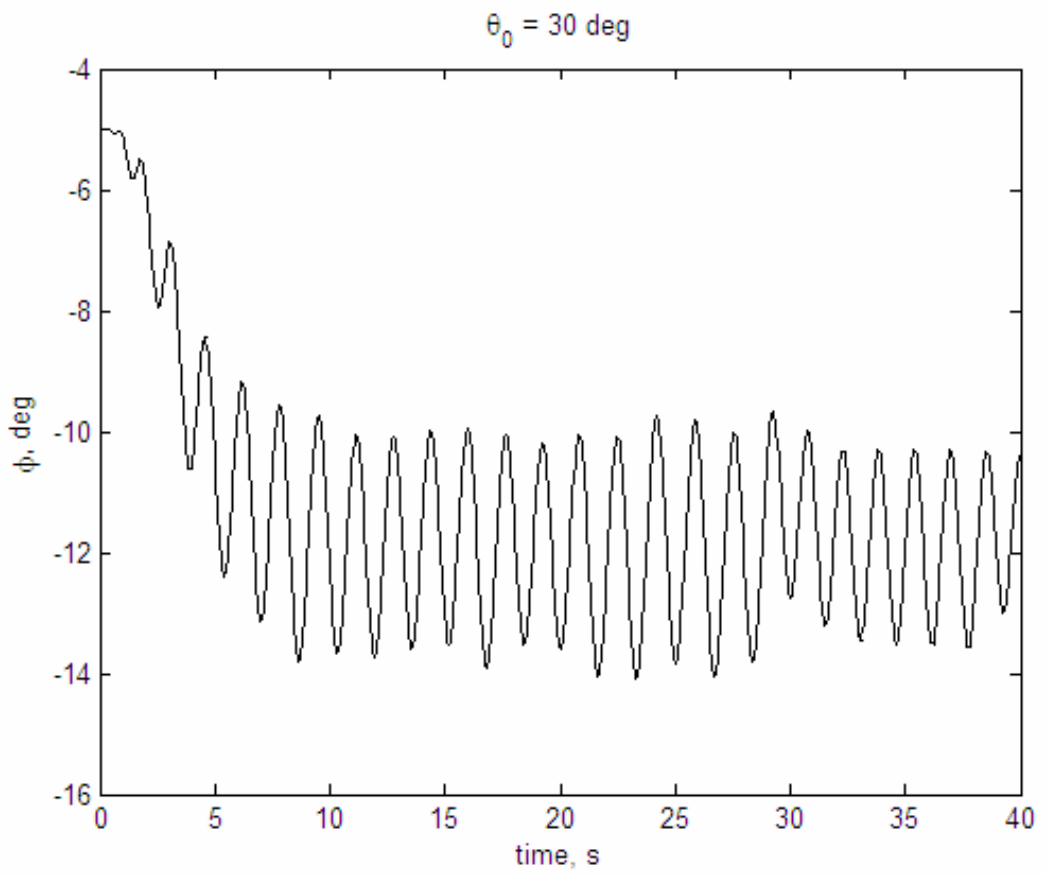

Figure 8. Free to roll simulations at wing pitch angle equal $30 \mathrm{deg}$, starting at roll angle equal -5 degree. 


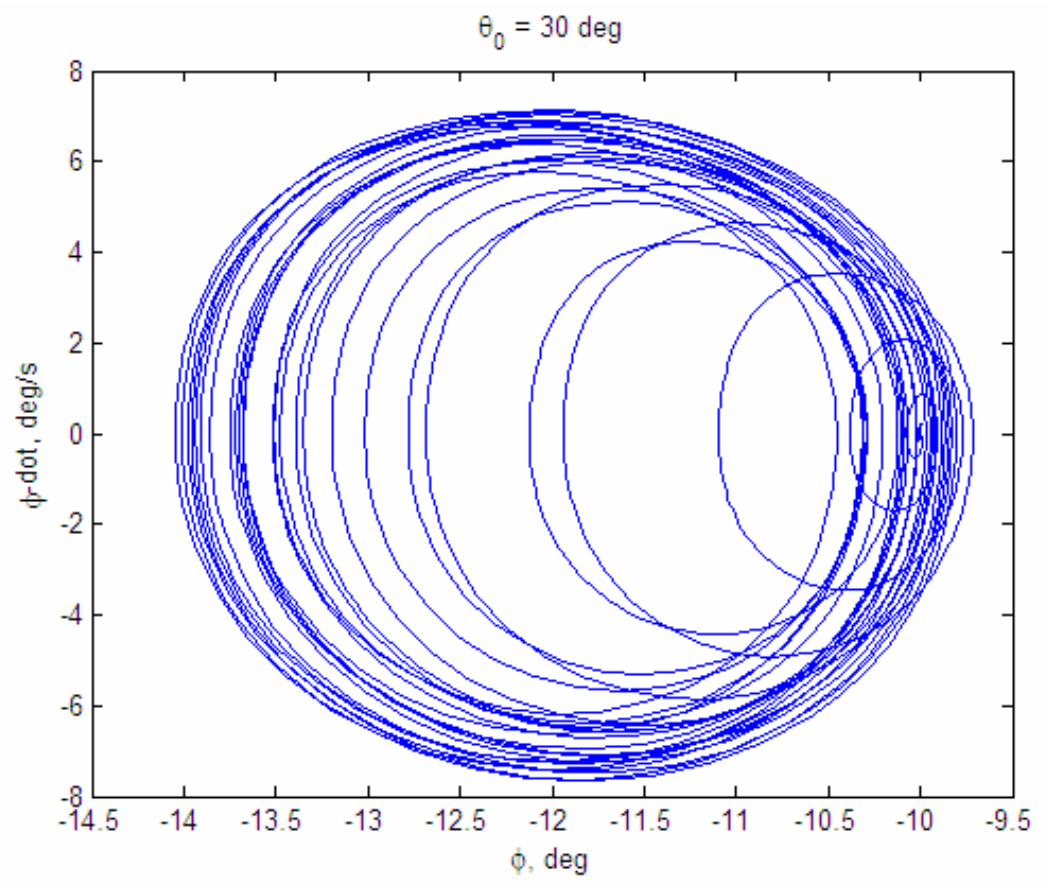

Figure 9. Phase-plane of the simulation response at pitch angle equal $30 \mathrm{deg}$, initial roll angle of -5 degree.

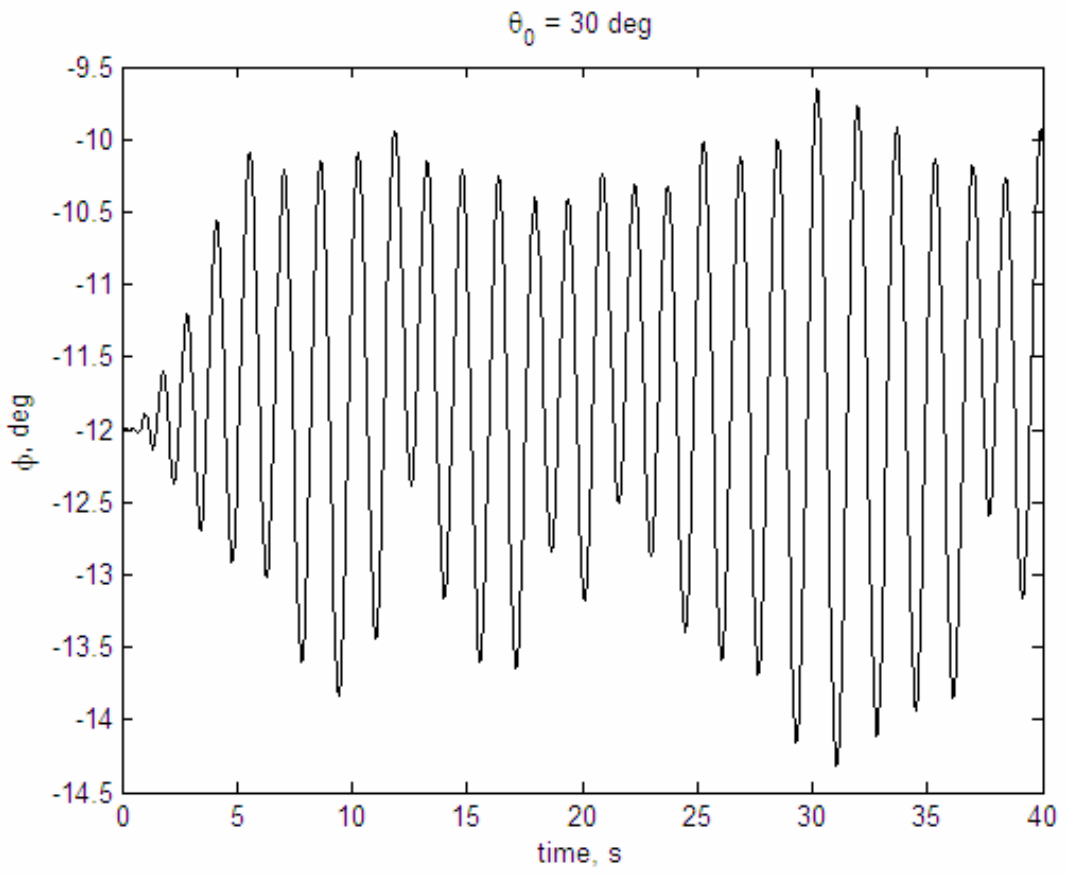

Figure 10. Free to roll simulations at wing pitch angle equal $30 \mathrm{deg}$, starting at roll angle equal -12 degree. 


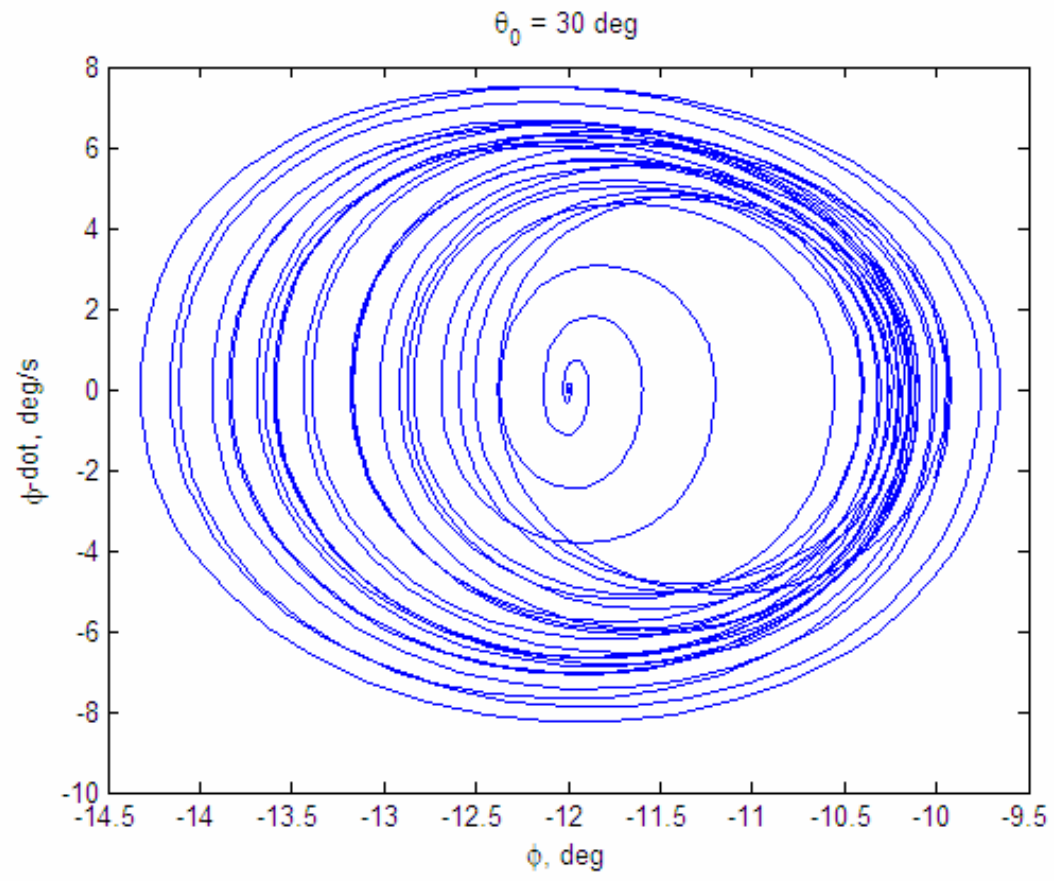

Figure 10. Phase-plane of the simulation response at pitch angle equal $30 \mathrm{deg}$, initial roll angle of -12 degree. 